\title{
EVALUATION OF OSTEOPATHY IN PATIENTS WITH BETA-THALASSEMIA MAJOR USING DIFFERENT IRON CHELATION THERAPIES
}

\author{
ALI JALAL SHAWKAT ${ }^{1 *}$, AHMED HAMED JWAID ${ }^{2}$, GHADA MARZOUQ AWAD ${ }^{3}$, HAYDER ADNAN FAWZI ${ }^{4}$ \\ ${ }^{1}$ Department of Clinical Pharmacy, Ministry of Health, Iraq. ${ }^{2}$ Department of Pharmacology, College of Pharmacy, University of Baghdad, \\ Baghdad, Iraq. ${ }^{3}$ Hereditary Blood Disorders Centre, AL-Karama Teaching Hospital, Baghdad, Iraq. ${ }^{4}$ Clinical Pharmacist, Baghdad Medical \\ City, Baghdad, Iraq. Email: alijalal0135@gmail.com \\ Received: 13 August 2018, Revised and Accepted: 10 October 2018
}

ABSTRACT

Objective: We aim to assess the bone mineral density (BMD) and bone biochemical parameters in Iraqi patients with $\beta$-thalassemia major ( $\beta$-TM).

Methods: Dual-energy X-ray absorptiometry scan was used to evaluate bone density and interpreted about Z-score which compares to the BMD of age-, sex-, and ethnicity-matched reference population. Biochemical parameters such as calcium, 25-OH Vitamin D, parathyroid hormone, and serum ferritin (SF) evaluated.

Results: No statistical difference in SF between pediatrics and adults was determined; however, 66 patients were having their SF between 1000 and $2500 \mathrm{ng} / \mathrm{ml}$ and 122 patients with SF > $2500 \mathrm{ng} / \mathrm{ml}$. Calcium and Vitamin D levels are low in both adults and pediatrics. The bone status shows high percentages of osteoporosis $62 \%$ and $54.5 \%$ for pediatrics and adults, respectively, as well as osteopenia $27 \%$ and $34.3 \%$ for both pediatric and adults and to a lesser extent normal bone status $11 \%$ for each.

Conclusion: Osteopathy has a high prevalence in Iraqi patients with $\beta$-TM and should receive an optimal transfusion and chelation therapy to prevent bone expansion. Calcium and Vitamin D should be routinely determined to prevent deficiency.

Keywords: Beta-thalassemia major, Osteoporosis, Osteopenia, Deferoxamine, Parathyroid.

(C) 2018 The Authors. Published by Innovare Academic Sciences Pvt Ltd. This is an open access article under the CC BY license (http://creativecommons. org/licenses/by/4. 0/) DOI: http://dx.doi.org/10.22159/ajpcr.2018.v11i11.29079

\section{INTRODUCTION}

Beta-thalassemia syndrome is a group of Hereditary Blood Disorders that are mainly characterized by the reduction or absence of betaglobin chain synthesis, resulting in a reduction of hemoglobin in red blood cells (RBCs), decreased the production of RBCs and consequently anemia $[1,2]$. Beta-thalassemia usually inherited as an autosomal recessive disease, more than $200 \beta$-thalassemia mutations have been identified, in some of which severe anemia is produced [3]. In Iraq, there is a high prevalence of $\beta$-thalassemia major ( $\beta$-TM) (the prevalence rate in 2015 was $27.4 / 100,000$ populations) [4]. $\beta$-TM refers to a severe phenotype which occurs when patients are homozygous or compounds heterozygous for $\beta$ chain mutation $(\beta+/ \beta 0, \beta 0 / \beta 0)$, patients commonly present with symptoms within the first 2 years of life $[5,6]$. The cornerstone management for patients with $\beta$-TM is based on lifelong transfusion and iron chelation [7]. Transfusion aims to correct anemia, suppress ineffective erythropoiesis and to inhibit gastrointestinal iron absorption [8]. Normally humans have no mechanism for excreting excess iron in iron overload conditions [9].

Physiologically two distinct cell types are in charge of the maintenance and renewal of the bone: The osteoblasts which are responsible for bone formation and the osteoclasts which are responsible for bone resorption and remodeling [10]. The essential pathway that links osteoclast-mediated bone resorption with osteoblast-mediated bone formation consists of a paracrine system that comprises receptor activator of nuclear factor- $\mathrm{\kappa} B$ ligand (RANKL), its RANK, and soluble protein osteoprotegerin (OPG). RANKL produced by osteoblasts and their precursors bind to the RANK receptor, promoting osteoclast differentiation and proliferation. OPG acts as a decoy receptor to block the action of RANKL. This system provides a balance between bone formation and resorption and through which a wide variety of biological mediators such as hormones, cytokines, and growth factors affect bone homeostasis. RANKL enhances osteoclastic function that elevated in $\beta$-TM patients, while OPG and OPG/RANKL ratio reduced, associated with low bone mineral density (BMD), this gives evidence that $\mathrm{OPG} /$ RANKL system plays a key role in the pathogenesis of osteoporosis in $\beta$-TM $[10,11]$.

Ineffective erythropoiesis results in erythroid hyperplasia and marrow expansion secondary to extramedullary hematopoiesis [6], resulting in expansion of the medulla, thinning of cortical bone and resorption of cancellous bone causing a generalized loss of BMD [11]. Deposition of iron in the bones impairs osteoid maturation and inhibits mineralization locally; this occurs by a mechanism that includes the incorporation of iron into calcium hydroxyapatite crystals which consequently affects their growth [12]. Deferoxamine (DFO) is an iron chelator used to reduce iron overload in patients with thalassemia, but on the other hand, it was found to have harmful effects on bone status. It exerts a direct effect by interfering with bone growth and by altering bone metabolism due to chelation of trace metals. DFO exerts deleterious effects on osteoblasts through inhibition of deoxyribonucleic acid (DNA) synthesis, osteoblasts proliferation, and differentiation of osteoblastic precursors and in patients receiving high doses it enhances osteoblasts apoptosis $[13,14]$.

Parathyroid hormone (PTH) secreted by the parathyroid gland and functions in calcium homeostasis together with Vitamin D and calcitonin, PTH maintains calcium levels within normal range by facilitating its absorption from the gastrointestinal tract, by phosphorous excretion and calcium reabsorption from the kidney, and by bone resorption. PTH also plays a role in the conversion of Vitamin D to its active form (1, 25-dihydroxycholecalciferol) in the kidneys [15].

Iron deposition within the parathyroid gland occurs in patients with iron overload due to recurrent transfusions and results in hypoparathyroidism (HPT), particularly after 10 years of age, as 
a consequence of that low PTH and Vitamin D ensued. HPT is also a leading cause of hypocalcemia, and several neurological complications may evolve such as tetany, seizures, and paresthesia $[16,17]$.

\section{METHODS}

\section{Patient selection and study design}

The present study designed as a single-center, cross-sectional, observational study. The study performed at AL-Karama Teaching Hospital/Department of Hereditary Blood Disorders (Thalassemia Center). The participant patients in this study were all with $\beta$-TM trait who were attending Thalassemia Centre at Al-Karama Hospital for recurrent blood transfusion and for receiving their iron chelation therapy. A total of 201 patients approved to enroll in the study, written informed consent taken from all patients (and for underaged patients the written informed consent also taken from their legal guardian). The patients divided into two main groups: Group A: Patients on DFO are subdivided into two groups: Children and adolescents (8-18 years) and adults (19 and more) and Group B: Patients on deferasirox (DFX) are subdivided into two groups: Children and adolescents (8-18 years) and adults (19 and more).

The sample size calculated using Raosoft sample calculator (online tool), a total of 600 patients with $\beta$-TM were registered at AL-Karama Thalassemia Centre; hence, a sample size of approximately 200 patients should be included assuming a margin of error of $5 \%$ and a confidence level of $95 \%$.

\section{Method of bone status evaluation}

About $5 \mathrm{ml}$ of fasting pre-transfusional venous blood collected and serum stored at $-20^{\circ} \mathrm{C}$ after separation. Serum ferritin (SF) level was measured using Cobas e 411 (Roche diagnostic equipment). The ELISA kits were used to determine serum levels of calcium and 25-OH Vitamin D. Immunoenzymatic assay used for the evaluation of PTH. Dual-energy X-ray absorptiometry (DEXA) scan of the lumbar spine region done as an index of bone density. BMD is expressed in grams of minerals per scanned square centimeters $\left(\mathrm{g} / \mathrm{cm}^{2}\right)$ and interpreted in relationship to Z-score which compares to the BMD of age, sex, and ethnicity of the matched reference population $[18,19]$. In the assessment of the lumbar spine, the region of interests placed on the L1-L4 vertebral bodies [20]. Patients with Z-score of -1SD or greater are considered normal, Z-scores between -1 and -2.5 are osteopenic (low bone mass), Z-scores of -2.5 or less are considered osteoporotic, and severely osteoporotic patients are those having Z-score of $<-2.5$ with fragility fracture [21].

\section{Statistical analysis}

Discrete variables presented using their numbers and percentages; Chisquare test was used to analyze the discrete variables (or Fisher exact test when Chi-square test is not valid; due to low sample size $<20$ and if two or more with an expected frequency is $<5$ ). Two samples t-test was used to analyze the differences in means between two groups (if both follow a normal distribution with no significant outlier). Binary logistic regression analysis was used to calculate the odds ratio and their $95 \%$ confidence intervals when the outcome can categorize into two binary levels, and if appropriate probability plot used to present the relationship. Linear regression analysis was performed to assess the relationship between different variables. If one or both of the variables were following a normal distribution, Pearson correlation is used. If both did not follow a normal distribution, the Spearman correlation used. Scatter plot was used to present the regression analysis. Correlation coefficient or standardized beta $(r)$ is a representative of magnitude and direction of the relationship; $0.00-0.29=$ little or no correlation; $0.3-0.49=$ weak; $0.5-0.69=$ moderate; $0.7-0.89=$ strong; and $0.9-1.00=$ very strong. The negative sign indicates an inverse relationship, while a positive sign indicates a direct relationship. Statistics is a Software Package 22 (Chicago, IL), Minitab 17.1.0 software package was used to make the statistical analysis, $\mathrm{p}$-value considered to be statistically significant if $<0.05$.

\section{RESULTS}

The demographic data and disease characteristics illustrated in Table 1; a total of 201 patients were approved to include in the study. In this study, the patients included fall into three groups: Children (8-12), $\mathrm{n}=40 ;(19.9 \%)$, adolescents $(13-18), \mathrm{n}=78 ;(38.8 \%)$, and adults $(>19)$, $\mathrm{n}=83 ;(41.3 \%)$, but children and adolescents were considered as a one category (pediatric) during the study. The frequency of their attendance was every 2 weeks, and their age of onset on blood transfusion was approximately $13.7 \pm 9.0$ months.

Although there is no statistical difference in SF between pediatrics and adults, 66 patients were having their SF between 1000 and $2500 \mathrm{ng} / \mathrm{ml}$ and 122 patients with SF $>2500$. Calcium and Vitamin D levels are low in both adults and pediatrics. The bone status shows high percentages of osteoporosis $62 \%$ and $54.5 \%$ for both pediatrics and adults, respectively, as well as osteopenia $27 \%$ and $34.3 \%$ for both pediatrics and adults and to a lesser extent normal bone status $11 \%$ for each as illustrated in Tables 2 and 3.

Advanced age predicts a decrease in BMD and osteoporosis insignificantly. However, males having higher BMD in comparison to females; there was no significant effect on BMD. DFX inversely associated with osteoporosis as illustrated in Table 4. Increase in iron overload predicts osteoporosis, but the relationship is insignificant.

In univariate analysis advance age, DFO and elevated ferritin predict osteoporosis (Table 5).

There was no significant correlation between the different variable in Table 6 with osteoporosis in the adult.

\section{DISCUSSION}

Osteopathy affects $40-50 \%$ of the patients with thalassemia major. The mechanism of osteopathy in $\beta$-TM is multifactorial and comprises the effects of anemia on bone health, secondary to iron deposition

Table 1: Demographic data and disease characteristics

\begin{tabular}{ll}
\hline Variables & Value \\
\hline Number & 201 \\
Age (years), mean \pm SD & $19.1 \pm 7.3$ \\
8-12 years & $40(19.9 \%)$ \\
13-18 years & $78(38.8 \%)$ \\
$\geq 19$ years & $83(41.3 \%)$ \\
Gender & \\
Female & $107(53.2 \%)$ \\
Male & $94(46.8 \%)$ \\
BMI $\left(\mathrm{kg} / \mathrm{m}^{2}\right)$, mean $\pm S D$ & $21.4 \pm 4.0$ \\
The frequency of attendance (days), mean $\pm \mathrm{SD}$ & $14.7 \pm 4.3$ \\
Age at which disease diagnosed (months), mean $\pm \mathrm{SD}$ & $13.7 \pm 9.0$ \\
\hline
\end{tabular}

SD: Standard deviation

Table 2: Assessment of bone status and various biochemical parameters

\begin{tabular}{llll}
\hline Variables & Paediatrics* & Adults & p-value \\
\hline Number & 100 & 101 & - \\
Ferritin (ng/mL) & $3,957.9 \pm 2,914.0$ & $3,761.7 \pm 2,452.9$ & 0.606 \\
$<1000$ & $7(7.0 \%)$ & $6(5.9 \%)$ & 0.737 \\
$1000-2500$ & $35(35.0 \%)$ & $31(30.7 \%)$ & \\
$>2500$ & $58(58.0 \%)$ & $64(63.4 \%)$ & \\
Calcium (mmol/L) & $2.06 \pm 0.23$ & $2.1 \pm 0.2$ & 0.227 \\
Vitamin D (ng/mL) & $10.5 \pm 8.5$ & $11.4 \pm 9.1$ & 0.498 \\
PTH (pg/mL) & $35.7 \pm 27.1$ & $34.0 \pm 23.4$ & 0.644 \\
Z score & $-2.7 \pm 1.4$ & $-2.5 \pm 1.2$ & 0.186 \\
Bone status & & & \\
$\quad$ Normal (>1 SD) & $11(11.0 \%)$ & $11(11.1 \%)$ & 0.509 \\
$\quad$ Osteopenia & $27(27.0 \%)$ & $34(34.3 \%)$ & \\
$\quad(-1)-(-2.5 \mathrm{SD})$ & & & \\
$\quad$ Osteoporosis & $62(62.0 \%)$ & $54(54.5 \%)$ & \\
$(<-2.5$ SD) & & & \\
\hline
\end{tabular}

*Pediatrics (children and adolescents). PTH: Parathyroid hormone 
(hemosiderosis) of various endocrine glands, liver dysfunction, chronic illness affecting physical health, and chelation therapy inducing toxicity. Recently, the receptor activator of nuclear kB (RANK)/RANK ligand and OPG system has been found to be the dominant pathway affecting osteoclastic activation and proliferation and plays a major role in thalassemia-induced osteoporosis [22,23]

DEXA is a non-invasive method for the assessment of BMD; in the present study, the osteopenia was in $27 \%$ of pediatrics and $34.3 \%$ of adults (totally $30.35 \%$ ), while osteoporosis was $62 \%$ in pediatrics and $54.5 \%$ in adults (totally 57.7\%), and the total normal BMD was in only $10.9 \%$. The results of the $\mathrm{Z}$ score in our study were comparable to Valizadeh et al. [19] and Merchant et al. [24]. Our findings state that there is no significant difference in $\mathrm{Z}$ score between adults and pediatrics which appears to be in sharp contrast to Mohseni et al. [25], in which they concluded that low BMD in pediatrics is less common than adults. However, in our study, there was a non-significant direct relationship between advancing in age and decrease in BMD and osteoporosis this was in agreement with AL-Amir et al. [26]; however, in pediatrics advance in age significantly (in univariate analysis) and dependently (in multivariate analysis) predict osteoporosis which

Table 3: SF levels and bone status among patients using DFO and DFX

\begin{tabular}{llll}
\hline Variables & DFO & DFX & p-value \\
\hline Number & 99 & 102 & - \\
Ferritin & $5028.8 \pm 3006.5$ & $2724.1 \pm 1699.2$ & $<0.001[\mathrm{~S}]$. \\
$<1000$ & $3(3.0 \%)$ & $10(9.8 \%)$ & $<0.001[\mathrm{~S}]$. \\
$1000-2500$ & $19(19.2 \%)$ & $47(46.1 \%)$ & \\
$>2500$ & $77(77.8 \%)$ & $45(44.1 \%)$ & \\
Z score & $-2.8 \pm 1.2$ & $-2.4 \pm 1.4$ & $0.014[\mathrm{~S}]$. \\
Bone status & & & \\
$\quad$ Normal & $5(5.1 \%)$ & $17(16.8 \%)$ & $0.009[\mathrm{~S}]$. \\
$\quad$ Osteopenia & $27(27.6 \%)$ & $34(33.7 \%)$ & \\
$\quad$ Osteoporosis & $66(67.3 \%)$ & $50(49.5 \%)$ & \\
\hline DFO: Deferoxamine, DFX: Deferasirox & &
\end{tabular}

Table 4: Predictors of osteoporosis for all patients

\begin{tabular}{lll}
\hline Variables & \multicolumn{2}{l}{ Osteoporosis } \\
\cline { 2 - 3 } & OR (95\% CI) & p-value \\
\hline Age & $1.009(0.971-1049)$ & 0.647 \\
Gender & $1.420(0.805-2.504)$ & 0.226 \\
BMI & $0.960(0.894-1.030)$ & 0.253 \\
Treatment (DFX) & $0.475(0.268-0.845)$ & $0.011[\mathrm{~S}]$. \\
Splenectomy & $2.083(0.970-4.470)$ & 0.060 \\
Ferritin & $1.000(1.000-1.000)$ & 0.149 \\
Calcium & $0.102(0.964-1.260)$ & 0.156 \\
Vitamin D & $0.974(0.943-1.006)$ & 0.113 \\
PTH & $1.010(0.998-1.023)$ & 0.109 \\
\hline
\end{tabular}

OR: Odds ratio, CI: Confidence interval, BMI: Body mass index, PTH: Parathyroid hormone, DFX: Deferasirox is in agreement with Merchant et al. [24] and Izadyar et al. [27] who reported a statistically significant relationship between age and BMD; nevertheless, Izadyar et al. have the same findings regarding gender in which both of us found no significant association between gender and BMD, and our finding regarding gender was in disagreement with Mohseni et al., who reported a significant effect of gender on BMD in which males had higher BMD, but their findings undermined because of its ineffectiveness on $\mathrm{Z}$ score [25].

The effect of splenectomy on BMD was not significant $(\mathrm{p}=0.06)$ which was in agreement with Merchant et al. [24] up to our knowledge, the effect of splenectomy on BMD was not studied more often. In adults, the increase in SF predicts insignificantly $(p=0.544)$ osteoporosis while in pediatrics $\mathrm{SF}$ is a dependent and significant predictor of osteoporosis, this was consistent with a study conducted on 70 children and adolescents by Nesheli and Farahanian [28] which reported a significant relationship between SF and bone density. The lack of significant correlation between SF and BMD in adults could attribute to SF tolerance, SF measurements may vary at different times, and other possibilities are small sample size, measurements error, and type of chelator used. The mechanism of iron toxicity on the bone is elucidated by its local effect which impairs osteoid maturation and bone mineralization which occurs by iron incorporation into calcium hydroxyapatite crystals which are important information of normal bones in which these crystals deposited on a proteinaceous collagen matrix [12].

DFO is considered a significant predictor for osteopathy, osteopenia and osteoporosis were higher in patients using DFO, and this could attribute to low compliance with DFO and high SF levels in these patients. DFO exerts a deleterious effect on bone through its direct effect by altering bone metabolism by chelating trace metals. DFO affects osteoblasts through inhibition of DNA synthesis, osteoblasts proliferation, and differentiation of osteoblastic precursors and in patients receiving high doses it enhances osteoblasts apoptosis [13]

The reason behinds low BMD and high prevalence of osteoporosis in both populations in the present study attributed to high SF levels, low calcium and Vitamin D levels, poor nutritional supports, limited physical activity, and inappropriate chelation. The impaired calcium hemostasis is thought to be a consequence of iron overload, Vitamin D deficiency, and HPT [29]. According to Shah study [30], hypocalcemia in states of iron overload is usually chronic and asymptomatic. Hypocalcemia (resulting from HPT) occurs through increasing calcium loss through urine and decrease intestinal absorption of calcium. The reported calcium levels in Salva's study were comparable to our levels.

The PTH levels in this study were various range from 8.6 to $62.8 \mathrm{pg} / \mathrm{ml}$ in pediatrics and from 10.6 to 57.4 in adults, the PTH levels in our study are comparable to many studies [26,31]. The development of HPT is mainly considered a consequence of iron overload, Pirinççioğlu and Söker [31] found a positive correlation between SF levels and PTH, while Chern and Lin [32] found that the development of HPT is not correlated with SF as many patients developed HPT while their SF

Table 5: Predictors of osteoporosis for pediatric patients

\begin{tabular}{|c|c|c|c|c|}
\hline \multirow[t]{2}{*}{ Variables } & \multicolumn{2}{|l|}{ Univariate } & \multicolumn{2}{|l|}{ Multivariate } \\
\hline & OR $(95 \% \mathrm{CI})$ & p-value & OR $(95 \% \mathrm{CI})$ & p-value \\
\hline Age & $1.169(1.034-1.320)$ & $0.012[\mathrm{~S}]$. & $1.090(0.919-1.293)$ & 0.31 \\
\hline Gender & $1.111(0.495-2.493)$ & 0.798 & - & - \\
\hline BMI & $0.959(0.870-1.057)$ & 0.398 & - & - \\
\hline Treatment (DFX) & $0.333(0.142-0.780)$ & $0.011[\mathrm{~S}]$. & $0.576(0.133-2.505)$ & 0.462 \\
\hline Ferritin & $1.000(1.000-1.000)$ & $0.022[\mathrm{~S}]$. & $1.000(1.000-1.000)$ & 0.459 \\
\hline Calcium & $1.091(0.943-1.262)$ & 0.240 & - & - \\
\hline Vitamin D & $0.987(0.941-1.035)$ & 0.581 & - & - \\
\hline PTH & $1.011(0.993-1.030)$ & 0.217 & - & - \\
\hline
\end{tabular}

$\mathrm{R}^{2}$ (Cox and Snell)=0.282, PTH: Parathyroid hormone, BMI: body mass index, DFX: Deferasirox, OR: Odds ratio, CI: Confidence interval 
Table 6: Predictors of osteoporosis for adult patients

\begin{tabular}{lll}
\hline Variables & \multicolumn{2}{l}{ Osteoporosis } \\
\cline { 2 - 3 } & OR (95\% CI) & p-value \\
\hline Age & $1.029(0.957-1.107)$ & 0.440 \\
Gender & $1.774(0.793-3.968)$ & 0.163 \\
BMI & $0.967(0.871-1.073)$ & 0.523 \\
Treatment (DFX) & $0.640(0.289-1.419)$ & 0.272 \\
Splenectomy & $1.818(0.757-4.366)$ & 0.181 \\
Ferritin & $1.000(1.000-1.000)$ & 0.544 \\
Calcium & $0.493(0.079-3.090)$ & 0.450 \\
Vitamin D & $0.964(0.922-1.009)$ & 0.118 \\
PTH & $1.009(0.991-1.026)$ & 0.326 \\
\hline
\end{tabular}

PTH: Parathyroid hormone, BMI: body mass index, DFX: Deferasirox, OR: Odds ratio, $\mathrm{CI}$ : Confidence interval

levels were lower than other patients. HPT development in their study was found to be dependent on patients' related factors and tendencies. Several mechanisms were postulated to describe the glandular damage by iron overload which mainly occurs in the second or third decade of life: 1 - the formation of free radicals and lipid peroxidation causing mitochondrial, lysosomal, and sarcolemmal membrane damage, 2 - number of surface transferrin receptors in the cell, and 3 - the ability of the cell to protect itself against iron species $[31,33]$.

Vitamin D is crucial for calcium and phosphorous absorption. Vitamin $\mathrm{D}$ has a direct relation to BMD, and maximum BMD achieved when the 25-OH-D level is $40 \mathrm{ng} / \mathrm{ml}$ or more [34]. In our study, serum Vitamin D levels are apparently deficient in both pediatrics and adults $(10.5 \pm 8.5 \mathrm{in}$ pediatrics and $11.4 \pm 9.1$ in adults) which are comparable to Akhouri and Neha [35] and Merchant et al. [24] Vitamin D deficiency in patients with $\beta$-TM attributed to nutritional deficiency and hepatic impairment due to hemosiderosis which results in defective hydroxylation of Vitamin $D$ to 25-OH-Vitamin D, levels of Vitamin D $>30 \mathrm{ng} / \mathrm{ml}$ is considered normal and levels between $10-20 \mathrm{ng} / \mathrm{ml}$ are considered deficient and levels of $21-29 \mathrm{ng} / \mathrm{ml}$ is considered insufficient [24,34].

\section{CONCLUSION}

Osteopathy (osteopenia and osteoporosis) has a high prevalence in Iraqi patients with $\beta$-TM. Poor chelation, undernourishment, calcium and Vitamin D deficiency, and secondary HPT are the leading cause of osteopathy. Therapeutic intervention should initiate in the case of Vitamin D and calcium deficiency. Annual BMD evaluation is crucial to avoid osteopathy in thalassemia patients in Iraq.

\section{ACKNOWLEDGMENT}

The authors would like to thank Al-Karama Teaching Hospital and Hereditary Blood Disorders employees, especially Dr. Osamah F. Jameel and Dr. Ahmad Jamalfor their big help and support throughout the research period. We are appreciative of Dr. Sarmad S. Hussein for his help in accomplishing the research.

\section{AUTHORS' CONTRIBUTION}

All authors contribute equally in the making of this manuscript.

\section{CONFLICTS OF INTEREST}

The authors declare that there are no conflicts of interest.

\section{REFERENCES}

1. Marengo-Rowe AJ. The thalassemias and related disorders. Proc (Bayl Univ Med Cent) 2007;20:27-31.

2. Devarshi S, James S, Najafzadeh E, Pawar S, Kalrao V, Bafna V. Assessment of quality of life, complications and post-transfusion adverse reactions in thalassemia pediatric in tertiary care hospital. Int $\mathrm{J}$ Pharm Pharm Sci 2016;8:317-20.

3. Muncie HL, Jr., Campbell J. Alpha and beta thalassemia. Am Fam Physician 2009;80:339-44
4. Kadhim KA, Baldawi KH, Lami FH. Prevalence, incidence, trend, and complications of thalassemia in Iraq. Hemoglobin 2017;41:164-8.

5. Jha R, Jha S. Beta thalassemia-a review. J Pathol Nepal 2014;4:633-71.

6. Aydinok Y. Thalassemia. Hematology 2012;17 Suppl 1:S28-31.

7. Tyagi P, Kumar Y, Gupta D, Singh H, Kumar A. Therapeutic advancements in management of iron overload-a review. Int J Pharm Pharm Sci 2015;7:35-44.

8. Makroo RN, Bhatia A. Provision of ideal transfusion support-the essence of thalassemia care. Apollo Med 2014;11:184-90.

9. Fuqua BK, Vulpe CD, Anderson GJ. Intestinal iron absorption. J Trace Elem Med Biol 2012;26:115-9.

10. Toumba M, Skordis N. Osteoporosis syndrome in thalassaemia major: An overview. J Osteoporos 2010;2010:537673.

11. De Sanctis V, Soliman AT, Elsefdy H, Soliman N, Bedair E, Fiscina B, et al. Bone disease in $\beta$ thalassemia patients: Past, present and future perspectives. Metabolism 2018;80:66-79.

12. Terpos E, Voskaridou E. Treatment options for thalassemia patients with osteoporosis. Ann N Y Acad Sci 2010;1202:237-43.

13. Zainal AA. Biochemical bone profile in thalassemia major patients on desferrioxamine therapy. Tikrit Med J 2010;16:122-8.

14. Alizzi FJ, Abbas WA, Fawzi HA. Assessment of the role of cholecystokinin in hyperemesis gravidarum and correlation with its severity. J Pharm Sci Res 2018;10:272-5.

15. Rofinda AZ, Arb F. Vitamin D and parathyroid hormone levels and their relation to serum ferritin levels in children with thalassemia major: Onecenter study in western Indonesia. J Adv Med Pharm Sci 2017;15:1-5.

16. Goyal M, Abrol P, Lal H. Parathyroid and calcium status in patients with thalassemia. Indian J Clin Biochem 2010;25:385-7.

17. Angelopoulos NG, Goula A, Rombopoulos G, Kaltzidou V, Katounda E, Kaltsas D, et al. Hypoparathyroidism in transfusion-dependent patients with beta-thalassemia. J Bone Miner Metab 2006;24:138-45.

18. Cosman F, de Beur SJ, LeBoff MS, Lewiecki EM, Tanner B, Randall S, et al. Clinician's guide to prevention and treatment of osteoporosis. Osteoporos Int 2014;25:2359-81.

19. Valizadeh N, Farrokhi F, Alinejad V, Said Mardani S, Valizadeh N, Hejazi S, et al. Bone density in transfusion dependent thalassemia patients in urmia, iran. Iran J Ped Hematol Oncol 2014;4:68-71.

20. Lorente Ramos RM, Azpeitia Armán J, Arévalo Galeano N, Muñoz Hernández A, García Gómez JM, Gredilla Molinero J, et al. Dual energy X-ray absorptimetry: Fundamentals, methodology, and clinical applications. Radiologia 2012;54:410-23.

21. Pirinccioglu AG, Akpolat V, Koksal O, Haspolat K, Soker M. Bone mineral density in children with beta-thalassemia major in Diyarbakir. Bone 2011:49:819-23.

22. Rossi F, Perrotta S, Bellini G, Luongo L, Tortora C, Siniscalco D, et al. Iron overload causes osteoporosis in thalassemia major patients through interaction with transient receptor potential vanilloid type 1 (TRPV1) channels. Haematologica 2014;99:1876-84.

23. Perisano C, Marzetti E, Spinelli MS, Calla CA, Graci C, Maccauro G. Physiopathology of bone modifications in beta-thalassemia. Anemia 2012;2012:320737.

24. Merchant R, Udani A, Puri V, D'Cruz V, Patkar D, Karkera A. Evaluation of osteopathy in thalassemia by bone mineral densitometry and biochemical indices. Indian J Pediatr 2010;77:987-91.

25. Mohseni F, Mohajeri-Tehrani M, Larijani B, Hamidi Z. Bone density changes in thalassaemic patients with age and time period. HK J Paediatr 2016;21:257-61.

26. Amir MA, Abdeen HM, Ezzat GM, Ghanem AA, Hassan AA. Predictors of osteopathy among adult patients with thalassemia major. Asian J Med Health 2017:5:1-9.

27. Izadyar S, Fazeli M, Izadyar M, Salamati P, Gholamrezanezhad A. Bone mineral density in adult patients with major thalassaemia: Our experience and a brief review of the literature. Endokrynol Pol 2012;63:264-9.

28. Nesheli HM, Farahanian E. Relation between bone mineral density and serum ferritin levels in patients with thalassemia major. Caspian $\mathrm{J}$ Pediatr 2016;2:158-63

29. De Sanctis V, Soliman AT, Elsedfy H, Yassin M, Canatan D, Kilinc Y, et al. Osteoporosis in thalassemia major: An update and the I-CET 2013 recommendations for surveillance and treatment. Pediatr Endocrinol Rev 2013;11:167-80.

30. Shah S. Assessment of serum calcium and phosphorus levels among transfusion-dependent beta thalassemia major patients on chelation therapy. J Postgrad Med Inst 2015;29:168-71.

31. Pirinççioğlu1 AG, Söker DG. Parathyroid functions in thalassemia major patients. Ann Clin Endocrinol Metab 2017;1:15-9.

32. Chern JP, Lin KH. Hypoparathyroidism in transfusion-dependent 
patients with beta-thalassemia. J Pediatr Hematol Oncol 2002;24:291-3.

33. De Sanctis V, Vullo C, Bagni B, Chiccoli L. Hypoparathyroidism in beta-thalassemia major. Clinical and laboratory observations in 24 patients. Acta Haematol 1992;88:105-8.
34. Soliman A, De Sanctis V, Yassin M. Vitamin D status in thalassemia major: An update. Mediterr J Hematol Infect Dis 2013;5:e2013057.

35. Akhouri MR, Neha D. Assessment of Vitamin D status and growth parameters in thalassemia major patients. J Dent Med Sci 2017;16:57-60. 